\title{
7. Calculating Networks: From Sociometry to PageRank
}

\begin{abstract}
This chapter ventures into the field of network algorithms, using the prehistory of Google's PageRank algorithm to discuss yet another way to think about information ordering. The chapter shows how algorithmic ordering techniques exploit and integrate knowledge from areas other than information retrieval - in particular the social sciences and citation analysis - and demonstrates how the 'politics' of an algorithm can depend on small variations that lead to radically different outcomes. The context of web search means that the various techniques covered in the second part of the book are brought together into a shared application space, allowing for a more concrete return to earlier discussions of variation and combination in software.
\end{abstract}

Keywords: PageRank, recursive status index, graph theory, sociometry, citation analysis

While many of the algorithmic techniques behind ordering gestures such as ranking, filtering, or recommending have indeed been pioneered in the context of information retrieval, there are other sites of inception that inform technical and conceptual trajectories. This chapter traces the development of network algorithms and the application of graph theory to information ordering through the fields of sociometry and citation analysis and then explains how these elements made their way into information retrieval, becoming part of Google's emblematic search engine.

Network analysis has seen a stellar rise over the last two decades: network visualizations have become a common sight in and beyond academia, social network analysis has found its way into the core curriculum of the social sciences, and certain scholars have gone as far as to declare the advent of a 'new science of networks' (Barabási, 2002; Watts, 2004). Powerful but

Rieder, B., Engines of Order: A Mechanology of Algorithmic Techniques. Amsterdam: Amsterdam University Press, 2020 DOI 10.5117/978946298619o_CHO7 
easy-to-use software packages such as Gephi (Bastian, Heymann, and Jacomy, 2009) have been highly successful in promoting network analysis, making the approach a mainstay in areas ranging from epidemiology to marketing. These tools incorporate sophisticated forms of calculation and visualization, and they have popularized concepts and techniques from graph theory beyond specialist circles. They enable and facilitate the analysis and mapping of relationships between websites using hyperlinks, between user accounts or content items on social media, or between words that co-occur in the same tweet or sentence. This popularity of network analysis as analytical tool has made it easier to talk about other domains where algorithmic techniques based on graph theory have proliferated.

Indeed, online platforms and services employ similar techniques to differentiate, rank, filter, connect, or group information items. A social scientist may use a network metric to identify 'opinion leaders' or to investigate power structures in a population of human beings more generally, but a social media site may use the exact same technique to decide which accounts to promote or which messages to discard from a content stream. While machine learning techniques are particularly competent at performing fast and self-adapting classification tasks and the relational database model is well suited for making complex and precise enquiries into data collections, network algorithms are especially apt when it comes to differentiating entities and ensembles in large-scale interaction systems. Unlike the empirical techniques discussed in the previous two chapters, which establish and operate on distributions or vectors describing objects and their properties, they channel descriptions and formalizations of pairwise relations between objects into another important intermediate form. In the context of web search, for example, a network perspective moves the focus of attention from the content of documents to the hyperlinks between them. This precise shift sits at the heart of the story behind Google Search, the company's web search engine, whose success is often attributed to PageRank, the most famous of all network algorithms and the focus of this chapter.

Using the algorithm's prehistory as an entry point into the large and complex field of network analysis, I will focus on what could be called 'evaluative metrics', techniques that, once again, seek to assess culturally embedded notions such as importance, relevance, authority, or quality through iterative calculations that attribute a value to each of the items under consideration. These metrics have come to play a significant role in a growing number of settings, and Google Search is just the tip of a much larger iceberg. 
Even if this chapter takes a particular algorithm and its self-proclaimed mission of 'bringing order to the web' (Page et al., 1999) as its telos and spends more time on conceptual embeddings, I will still emphasize variation and technical coupling over singular logics and paradigmatic purity. Focusing on PageRank has the advantage of dealing with a contemporary algorithm that is well documented in two research papers (Brin and Page, 1998; Page et al., 1999) as well as two US patents (Page 2001, 2004) that, remarkably, are more thorough in their citation practices than the academic publications. Additionally, RageRank is one of the few algorithms venturing into deeply mathematical territory that has not only been studied extensively by mathematicians and computer scientists (Langville and Meyer, 2004; Bianchini, Gori, and Scarselli, 2005), but is also regularly commented on by scholars in the humanities and social sciences (Introna, 2007; Diaz, 2008; Rieder, 2009). This relative familiarity with the concept and its application in web search will hopefully make certain aspects of my argument more vivid and provide grounding material for the more abstract technicities I will address.

This chapter relates more explicitly to contemporary fields such as 'web search studies' (Zimmer, 2010) than earlier ones, but the intellectual genealogy of the models and techniques in question here can be traced back to at least the 1930s. It leads to another important trading zone, one that primarily involves mathematics and the social sciences but remains largely distinct from the well-documented history of statistics (Hacking, 1990; Stigler, 1999). In the first part of this chapter, I will reconstruct the historical context, focusing on the particular idea that one can measure the 'authority' of a node in a (social) network recursively, that is, by taking into account not only the number of connected nodes but also their respective status. ${ }^{1}$ This idea has often been presented as a core innovation in PageRank: when calculating the 'importance' of a web page, a link from a highly ranked site is 'worth' more than a link from a site with a low rank. The second part of the chapter examines PageRank more concretely, first by comparing it to the very similar HITS algorithm (Kleinberg, 1999), and second by closely scrutinizing the damping factor $\alpha$ with the help of the sociometric texts that first introduced such a parameter into recursive status calculations. The argument begins with a closer look at the history of graph theory and its close relationship with the social sciences.

1 For the wider historical and conceptual background of the methods discussed here, see Mayer (2009). 


\section{Social Theory, Graph Theory}

In the introduction to his Theorie der endlichen und unendlichen Graphen (Theory of finite and infinite graphs) from 1936, the first textbook on graph theory, the Hungarian mathematician Dénes Kőnig (1936) wrote that '[p]erhaps even more than to the contact between mankind and nature, graph theory owes to the contact of human beings between each other' (n.p.), pointing to the often overlooked fact that modern graph theory developed, perhaps even more so than statistics, in close contact with the social sciences. Rather than being imported into these disciplines as a ready-made mathematical toolkit, graph theory was shaped in no small part by the measuring fever in the field of 'small group' research, founded by Kurt Lewin and Jacob L. Moreno. Even the mathematician Frank Harary, the central figure in the development, application, and standardization of graph theory in the second half of the twentieth century, first came into contact with his future field of specialization at the Research Center for Group Dynamics, established by Lewin in 1948 at the University of Michigan (Harary, 1969, p. 6). This close relationship warrants further investigation.

\section{The Development of Graph Theory in Relation to the Social Sciences}

Despite its enormous success in recent decades, graph theory - 'a mathematical model for any system involving a binary ${ }^{2}$ relation' (Harary, 1969, p. v) - has historically not been considered a noble endeavor. Although prominent names such as Leonhard Euler, Gustav Kirchhoff, Arthur Cayley, and William Hamilton are attached to its history, it is only in the second half of the twentieth century that graph theory emerges from what J. H. C. Whitehead called the 'slums of topology' (cit. in Erdős, 1977, p. 3). The Journal of Graph Theory was founded only in 1977, which further testifies to a lack of interest from (pure) mathematicians. But besides the growing number of practical applications in chemistry, physics, and engineering, it is in the social sciences, and specifically in social psychology, that a problem space appears that proves particularly fertile for the development of a mathematics concerned with structure. Unlike the library problem', which is intrinsically tied to the pragmatics of institutions having a clear utilitarian mission, whether that mission is public education or the support for knowledge

2 In graph theory, a connection always runs between exactly two individual elements, with the exception of a 'loop', that is, a node that links to itself. 
work and decision-making, this particular space revolves around the more open-ended problems of scientific inquiry.

A first line of development can be traced to Jacob L. Moreno's (1934) book Who Shall Survive?, an original and idiosyncratic volume that launched the field of sociometry as 'the mathematical study of psychological properties of populations' (p. 432). Developed out of group psychotherapy, sociometry was essentially organized around the sociometric test, a questionnaire distributed in small- and mid-sized groups - schools, factories, etc. - that asked people to choose the individuals they liked best, admired most, had the most contact with, or similar questions. The resulting pairwise (network) data was thought to reveal the 'psychological structure of society' (p. 9) and was displayed and analyzed as a sociogram, a manually arranged point-and-line diagram that would nowadays be called a 'network visualization'. But while Moreno indeed spearheaded the visual display of the network data produced by the sociometric test, his 'mathematical study' was underdeveloped in terms of actual mathematical method, which prompted criticism 'for lack of the methodological stringency appropriate to science' (Mayer, 2009, p. 6o). Moreno's work still garnered significant interest and the journal Sociometry, founded in 1937, attracted a number of contributors with mathematical skill, who started to work on the peculiarly rich and complex data produced by a remarkably simple questionnaire.

In 1946, Forsyth and Katz made a critical step toward mathematization by proposing to replace the networks drawn in a process of trial and error with a square matrix, often referred to as 'adjacency matrix', in order to 'present sociometric data more objectively' (p. 341). While the goal was to display a graph ${ }^{3}$ in a way that was less confusing than the hand-drawn sociograms, the authors also produced a quasi-algorithmic technique to manipulate the matrix simply by reordering rows and columns in a way that subgroups would become visible. The shift in mode of representation also opened the door for the application of relatively simple but powerful methods from matrix algebra (Festinger, 1949).

3 While the terms 'network' and 'graph' are frequently used synonymously, it should be noted that mathematicians often make a distinction based on levels of abstraction and formalization: 'There are hierarchies of mathematical models. A network is a model for empirical flows. A graph or digraph is a model for a network - it serves as a first approximation to a network with numerical or other values on its edges (or arcs) and maybe on its vertices. For a graph or digraph captures the structure of a network while suppressing the additional numerical information' (Harary, 1983, p. 355). 


\begin{tabular}{lcccc}
\hline & node 1 & node 2 & node 3 & $\ldots$ \\
\hline node 1 & 0 & 1 & 0 & \\
node 2 & 1 & 0 & 1 & \\
node 3 & 0 & 1 & 0 & \\
$\ldots$ & & & \\
\hline
\end{tabular}

A generic adjacency matrix describing an (undirected) graph. This square matrix uses binary values, but numerical values are often used as well if the data includes some measure of frequency or intensity for the relations between nodes.

Just like statistics provides a number of (standardized) techniques, for example, to establish a correlation coefficient between two variables, the matrix constitutes an intermediate form that points directly toward different operations that rely on linear algebra to produce 'views' on sociometric data, structural measures that are essentially 'order from order' (Chun, 2011, p. 101ff.), transformations of numbers into other numbers. We should not make the mistake, however, to dismiss such calculations as tautological, as essentially expressing the same thing as the original input; each calculation reveals some aspect of the data. In this sense, they are further examples for mechanical reasoning, transformations that become 'interpretations', ways of reading and making sense of the world in terms that are simultaneously reductionist (phenomena are reduced to a point-and-line model) and endlessly generative (the number of possible transformations is potentially infinite). Although Gerard Salton's work dealt with a different problem space and used the somewhat different term-document matrices, it was able to draw on the same space of mathematical technique, highlighting one of many (possible) intersections between trajectories.

Furthermore, the matrix approach made it possible to connect the work on sociometric data to a second line of development in the mathematization of social structure. During the 1940s, Bavelas (1948) had developed an innovative sketch for the mathematical expression of group structures, building on Kurt Lewin's Principles of Topological Psychology (1936). Using mostly geometric methods, he introduced such concepts as 'geodesic distance' and 'centrality' into the social network vocabulary. Unlike notions of statistical similarity (or distance), these measures were built on the idea of 'stepping' from one 'cell' (node) to the next. The distance between two nodes is thus the number of steps it takes to get from one to the other. As Festinger argued, the matrix approach proved useful here, because it could be directly applied to 'such concepts as diameter of [topological structures] and distance from one part of the structure to another' (Festinger, 1949, p. 157). The shift from 
the point-and-line diagram to the matrix representation thus reinforced the idea that social structure could become fully calculable and encouraged the import of techniques from algebra and geometry.

According to Barnes and Harary, the 'first indisputable application of graph theory to network analysis did not come until 1953' (Barnes and Harary, 1983, p. 237) when Harary and Norman's short volume Graph Theory as a Mathematical Model in Social Science (1953) was published. It marks a shift in disciplinary background similar to what we have seen in information retrieval: so far, most of the attempts at constructing mathematical methods for expressing and analyzing sociometric data had come from sociologists and psychologists with an interest in mathematics - with the notable exception of the statistician Leo Katz. But this was the work of two mathematicians with an interest in the social sciences. Frank Harary in particular established himself as the key figure in the 'mathematization' of sociometry, even before the publication of his seminal Graph Theory (1969), which not only standardized concepts, vocabulary, and methods, but also presented them in a pedagogical, applicable way that was crucial for the spread of graph theory and associated techniques.

While the more rigorous mathematical approach did not mean that ties with the social sciences were severed - Harary regularly collaborated with social scientists throughout his long career - there were nonetheless three important consequences. First, pictorial representations of networks and visual forms of analysis faded into the background. Network diagrams were still used, but rather as teaching aids in textbooks than as research tools in empirical work. Only the spread of graphical user interfaces in the $1990 \mathrm{~s}$ and the development of layout algorithms, based for example, on force simulations, lead to a true renaissance of the now so familiar point-and-line diagrams. Second, network metrics and methods for mathematical analysis proliferated and became both conceptually and computationally more demanding, sometimes to a point where their empirical applicability became technically forbidding and methodologically questionable, even to scholars sympathetic to the general approach (cf. Granovetter, 1973, p. 1361). Third, although exchange between the mathematical methods of graph theory and empirical work in the social sciences has remained strong over the last decades, the movement toward abstraction and 'purification' implied by mathematization has led to a certain demarcation between the two. On the one side, graph theory has turned more and more into an 'immutable mobile' (Latour, 1986), an inscription that can travel from one setting to another without losing its shape. Stripped of the marks of their origin, these techniques - packaged in handbooks or software programs - are ready to be 
applied wherever a network can be found or formalized. On the other side, there has been a certain tendency, especially in less quantitative circles of sociology and social theory, to use the network model as an ontological concept rather than an analytical one, and to endow it with certain properties, for example, decentralization, uncontrollability, opposition to hierarchies, and so on. ${ }^{4}$ This is largely at odds with the epistemological commitment that underpins the application of graph theory to social phenomena, which is not a commitment to the network as a positive empirical entity but primarily to concepts and methods for studying empirical phenomena that can be modeled as networks. Consider the following quote:

As we have seen, the basic terms of digraph theory are point and line. Thus, if an appropriate coordination is made so that each entity of an empirical system is identified with a point and each relationship is identified with a line, then for all true statements about structural properties of the obtained digraph there are corresponding true statements about structural properties of the empirical system. (Harary, Norman, and Cartwright, 1965, p. 22)

What takes shape over these lines is a separation between the empirical and the analytical that is characteristic to quantitative empirical research and shifts the full epistemological weight onto the shoulders of 'formalization', that is, onto the 'appropriate coordination' between the two levels. I do not want to contest this separation in philosophical terms here, but rather put forward the much simpler critique that in order to produce a formalization of social phenomena that can be mapped unto the axioms of graph theory, a commitment has to be made, not to the network as an ontological category, but rather to a 'theory of the social' that supports and substantiates the formalization process, much like lay theories of language justified the representation of documents as term vectors in previous chapters. In Moreno's (1934) case, we find a theory of psychological 'attractions and repulsions' between 'social atoms' that bears the epistemological weight of formalization in the sense that it justifies the mapping of a relationship between two people onto two points and a line by conceiving 'the social' as a primarily dyadic affair.

4 This tendency has been particularly strong in media studies and adjacent fields where communication and computer networks proliferate. While these particular networks may indeed have very specific properties, graph theory does not imply a theory of the nature of empirical networks. This would seem obvious to a mathematician, but when reviewing three titles in media studies, Berry has to explicitly remind his readers that the 'network is not ontological it is analytical' (Berry, 2008, p. 365). 
Computerization conveniently transforms this process from a matter of theoretical elaboration into one of design. The grammatization of operational entities, which spans data models, program logic, and interface elements, indeed 'produces' atomized entities and their relations: social media services, to use a recognizable example, do not describe empirical systems, but fabricate them by building communicative infrastructures where user profiles interact with each other in a variety of ways. Just like early information retrieval pioneers were comfortable processing language without more than a rudimentary theory of language, the treatment of accounts as nodes and friendship connections as links requires little in terms of epistemological commitment. The 'appropriate coordination' mentioned above, which keeps social scientists awake at night, comes easy when digital objects are no longer formalizations of something else, but 'natively' (Rogers, 2013, p. 1) digital objects that are already fabricated axiomatic entities, conveniently transposable into basic intermediate forms. I will come back to this question further down, after having looked at the historic development of a specific type of measure that develops against the backdrop I have just outlined: the recursive status index.

\section{Recursive Status in Sociometry}

While social theorists (Castells, 1996; Benkler, 2006) have regularly opposed network forms of social organization to hierarchies over the last decades, the empirical-analytical approach followed by small-group research makes no such opposition; quite to the contrary, graph theory is seen as a means to detect and to measure social hierarchies of different kinds. As already mentioned, despite the definition of sociometry as mathematical study, Moreno's mathematical toolkit was rudimentary. But the sociometric test yielded such rich and unconventional data that even basic counting could lead to curious discoveries, for example, concerning the distribution of interpersonal attraction. When asking people whom they liked best, had the most contact with, or admired the most, the resulting distribution would be invariably skewed. Transposing such a 'choice distribution' from the New York State Training School for Girls, ${ }^{5}$ which Moreno used as his empirical playground, to New York City, he made an interesting observation:

For New York, with a population of $7,000,000$, the above percentages [taken from the Training School for Girls] would be after the $1^{\text {st }}$ choice, $3,200,000$

5 Gießmann (2009, p. 274) describes this reeducation camp for young girls, where Moreno worked, as a 'Dogville in the US of the 1930s'. 
individuals unchosen; after the $2^{\text {nd }}$ choice, 2,100,00o unchosen; after the $3^{\text {rd }}$ choice, 1,400,000 unchosen; after the $4^{\text {th }}$ choice, $1,200,000$ unchosen; and after the $5^{\text {th }}$ choice, 1,050,000 unchosen. These calculations suggest that mankind is divided not only into races and nations, religions and states, but into socionomic divisions. There is produced a socionomic hierarchy due to the differences in attraction of particular individuals and groups for other particular individuals and groups. (Moreno, 1934, p. 250f.)

The idea that social structure is hierarchic, even in the absence of explicit, institutional modes of hierarchical organization, and that a network approach can identify these stratifications is recognizable in this passage, 80 years before a 'new' science of networks (Watts, 2004) began to find power-law distributions for connectivity in most of the places it looked. But Moreno's 'socionomic hierarchy' is only the beginning of a long-lasting relationship between applied network mathematics and rankings of various kind. The search for a social pecking order can indeed be seen as the central goal of sociometry and from the 1950s onward, new techniques based on structural measures were introduced at a regular pace.

A formal analysis of differences in 'status', 'authority', and 'influence', the three terms used most frequently - and often interchangeably - in this context, developed in line with the matrix approach outlined above. One of the techniques Festinger (1949) put forward consisted of simply squaring a matrix (multiplying it by itself), which results in a new matrix where each entry shows the number of two-step connections between two nodes. Cubing the matrix would get the numbers for three step paths, and so on. If applied to data from a sociometric test asking participants to indicate the persons whose opinion they valued most, this technique would show 'who influences the greatest number of people in less than a specified number of steps' (Festinger, 1949, p. 156). The question of social power becomes a matter of calculation.

Here, a specific paper, cited in both PageRank patents, stands out: in 1953, Leo Katz published 'A New Status Index Derived from Sociometric Analysis' in the journal Psychometrika and introduced the notion of a 'recursive' or 'cumulative' status index. Building on his earlier work on matrix representations of data collected by Moreno (Forsyth and Katz, 1946), Katz proposed a 'new method of computation' (Katz, 1953, p. 39) for calculating social status. Status measures were already common in studies using the sociometric test, but they were essentially based on simple counting, as seen in Moreno's discussion of socionomic hierarchies. Katz explicitly rejected this method and argued that 'most serious investigators [...] have been dissatisfied with 
the ordinary indices of "status," of the popularity contest type' because this 'balloting' of 'votes' would ultimately not allow to 'pick out the real leaders' (p. 39). His goal was not to measure popularity but social power, even if the latter term was not explicitly used. Therefore, Katz proposed a new index that takes into account 'who chooses as well as how many choose' (p. 39), which means that votes from 'small fry' (p.42) would simply count less. This shift rests on the idea that status is cumulative in the sense that the topology of a social network reflects a latent socionomic hierarchy, to stick with Moreno's term, in which the status of an individual largely depends on the status of her network neighborhood. Who you are is who you know.

Katz proposes a technically simple but conceptually challenging technique that transforms the initial sociometric choice matrix by first computing all paths between all nodes, then attributing a lower weight to longer paths through 'damping', and finally calculating a metric for every node based on the combined weight of their connections to all other nodes (Katz, 1953; Hanneman and Riddle, 2005). An individual that can reach far into the network in only a few steps will thus receive a higher status number than an individual that has a lot of immediate connections that do not lead deeper into the network. Similar measures to what is today known as 'Katz centrality' abound in the following decades and they are not only used for status measuring but also for other things, for example, to identify cliques (Hubbell, 1965). The contribution by the mathematical sociologist Phillip Bonacich (1972) is worth noting, because it consists of a metric based on the eigenvectors of a matrix, which means that not all paths between nodes are taken into account like in Katz's case, but only the shortest paths. ${ }^{6}$ This makes the metric less dependent on local properties of networks and consequently more difficult to 'cheat' by adding nodes and connections locally. This resistance to what could be called 'link spam' is probably the reason why PageRank is a variant of eigenvector centrality rather than Katz centrality, although references to Bonacich's work are conspicuously absent from the patents.

\section{Citation Analysis}

A second arena where concrete techniques for the mathematical exploration and measurement of networks were pioneered - and directly applied to decision-making - is citation analysis, a field that shares a focus on scientific

6 For a deeper discussion of the various calculations and their differences, see Hanneman and Riddle (2005), Chapter 10. 
literature with information retrieval. In 1963, the Institute for Scientific Information, founded by Eugene Garfield, printed the first edition of the Science Citation Index (SCI), an index of citations from 613 journal volumes published in 1961, manually extracted and sorted by computer (Garfield, 1963). With the first edition storing already 1.4 million citations on magnetic tape, this index was perhaps the first 'big data' network file available in the social sciences and several researchers used it over the following years to test different computational methods. One of these methods illustrates the considerable generativity of the graph representation: Henry Small's co-citation technique creates a new network on top of the citation data by looking not at direct references, but at 'the frequency with which two documents are cited together' (Small, 1973, p. 265), that is, cited in the same article. The technique thus creates a graph with variable link strength, since two papers may be cited together an arbitrary number of times, introducing a level of nuance that the underlying data lacks. Although Small knew Gerard Salton's work and, indeed, proposed co-citation as a measure of 'subject similarity', the relationship between information retrieval and citation analysis remained hesitant. Salton himself was aware of Garfield's index (cf. Salton, 1963), but his own experiments with citation data did not draw on the SCI, which was conceived as a commercial product from the beginning.

Garfield's initial strategy (1955) was to promote the SCI first and foremost as an 'association-of-ideas index', a tool for finding scientific literature that addressed the same problem space as information retrieval and again presented itself explicitly as an alternative to traditional subject headings and classifications. But it quickly became obvious that a series of evaluative metrics could be derived from the SCI without much effort. Thus, in 1972, Garfield presented a fleshed-out version of a concept he had initially introduced as a tool for the historiographical study of science, the (in)famous 'impact factor', claiming that '[p]erhaps the most important application of citation analysis is in studies of science policy and research evaluation' (Garfield, 1972, p. 478). When taking into account that the first attempt at citation ranking by Gross and Gross (1927) was designed to help university libraries decide which chemical journals they should subscribe to, it becomes clear that any evaluative metric would instantly face considerable normative and institutional entanglement, much more so than the mostly descriptive sociometry.

Highly applicable to questions concerning resource allocation, citation analysis - and all of scientometrics for that matter - oscillates between disinterested forms of metrological realism and the accounting realism of science management. The impact factor and its many variants provide 
measures of scientific recognition or success that resonate perfectly with contemporary 'audit cultures' that 'present themselves as rational, objective and neutral, based on sound principles of efficient management' (Shore and Wright, 2000, p. 61). Even if the 'engine of order' concept focuses primarily on integrated forms of performativity that revolve around computerization, digital infrastructures, and the tight coupling of data, analysis, decision, and application, citation analysis is one of many settings where we can observe how other kinds of 'operational assemblages', such as bureaucracies, produce their own forms of ordering based on calculation. As Burrows remarks, the performative character of 'quantitative control' has reached the point where 'academic metric assemblages are at the cusp of being transformed from a set of measures able to mimic market processes to ones that are able to enact market processes' (Burrows, 2012, p. 368). Another example is a particularly unexpected and mildly insidious application of social network analysis for a very literal form of ordering or arrangement: researchers used students' Facebook friendship networks, retrieved with the Netvizz application (Rieder, 2013), to break up the seating order during university exams to minimize cheating - with some success, apparently (Topîrceanu, 2017). Engines of order can be built out of various materialities.

The impact factor, calculated for scientific journals, is an extremely basic measure: it takes the number of citations the last two volumes of a journal received the following year and divides it by the number of articles published in these two volumes. This division highlights an important question - and certainly a central reason why literature from the citation analysis field is cited in the PageRank patents - that is not addressed in sociometry, namely the problem of size. If a journal publishes a large number of articles it is well positioned to receive more citations than a journal that publishes fewer; purely counting citations without taking publication volume into account would therefore be misleading and this is why the impact factor divides by the number of articles published (Garfield, 1972).

A 1976 paper by Pinski and Narin pushed things significantly further by pointing out two problems with Garfield's measure. First, citations have equal value in the impact factor scheme, although 'it seems more reasonable to give higher weight to a citation from a prestigious journal than to a citation from a peripheral one' (p. 298). Pinski and Narin therefore proposed a recursive index for importance, based on the same eigenvector calculations we found in Bonacich's work, although the authors were apparently not aware of sociometric techniques. Second, Pinski and Narin argued that the impact factor attributed disproportional importance to review 
journals that are referenced a lot for convenience rather than scientific contribution, and 'can therefore not be used to establish a "pecking order" for journal prestige' (p. 298). Again, they propose a solution by including an 'input-output' measure into their calculations (p. 300) where the 'influence weight' (W) of incoming citations (C) is divided by the number of outgoing citations (S):

$$
W_{i}=\sum_{k=1}^{n} \frac{W_{k} C_{k i}}{S_{i}}
$$

This formula basically states that the 'influence weight' $(W)$ of a document should be the sum of the weight of the citations it receives, divided by the number of outgoing citations. If a document cites a lot of other sources ( $S=$ large), the 'value' it passes on to each of those sources will be lowered.

This means that the more papers an article cites, the lower the value 'transferred' to each of them. Review journals, which are not only cited a lot but also heavy citers themselves, are therefore no longer favored as brokers of influence. The exact same principle is at work in PageRank: the more outgoing links a site has, the lower the value of each link. This small twist shows how malleable these metrics are and how they react and adapt to new problems along the way. If we were to introduce a damping factor into this model, such as Katz's connection weight reduction scheme, in order to attenuate the 'free flow of citations in the referencing marketplace' (p. 298), we would essentially end up with PageRank, here in its canonical formulation:

$$
r(A)=\frac{\alpha}{N}+(1-\alpha)\left(\frac{r\left(B_{1}\right)}{\left|B_{1}\right|}+\ldots+\frac{r\left(B_{n}\right)}{\left|B_{n}\right|}\right)
$$

PageRank basically applies a damping factor a (more on that further down) to Pinski and Narin's formula: $r(A)$ is the weight to be calculated (equivalent of Wi in Pinski \& Narin), $r(B n)$ stands for the weight of incoming links ( $W k C k i$ above) and $|\mathrm{Bn}|$ for the number of outgoing links they have ( $\mathrm{Si}$ above).

While Pinski and Narin do not quote the work of economist Wassily Leontief, unlike Hubbell (1965), who acknowledges this source of inspiration in his sociometric clique identification scheme, there is reason to believe that their version of an input-output model was equally inspired by economic thinking, in particular if we take into account that Pinski and Narin's metric 
was intended for a 'funding agency with its need to allocate scarce resources' (Pinski and Narin, 1976, p. 312).

This is one of many instances where we can observe clear affinities between algorithmic information ordering and market models. In their analysis of the effects of both the computer and the associated information concept on the discipline of economics, Mirowski and Nik-Khah (2017) indeed argue that "what it meant to "know the truth" changed dramatically and irreversibly after World War II' (p. 1). They describe an epistemological nexus where concepts like 'equilibrium' or 'emergence' receive a decidedly computational interpretation: neoclassical economics, in particular, frame the market as 'the greatest information processor known to humankind' in the sense that it 'effectively winnows and validates the truth from a glut of information' ( $p .7)$. While this book cannot provide a comprehensive analysis of the complex relationship between modern economics and algorithmic information ordering, the integration of economic thinking into the conceptual horizon can also be observed in sociometry and this merits closer attention.

\section{From Sociometry to Social Exchange Theory}

While the sociometric test was generally lauded for producing valuable data, Moreno's socio-psychological theory of attraction and repulsion between social atoms (Moreno, 1934, p. 6) was based on contested psychoanalytical assumptions and his goal to develop a 'technique of freedom' that reorganizes society by training individuals to transcend their social prejudices in order to liberate the forces of 'spontaneous attraction' did not go well with the sober and pragmatic mindset of most empirical sociologists. The sociometric papers working with Moreno's data therefore mostly subscribed to a vague version of the same atomistic and dyadic view of society, which enabled and justified the point-and-line formalization needed to apply graph theoretical methods, but they shunned the deeper aspects of the theoretical horizon behind it. Concepts like influence, status, importance, prestige, and so on filled the void, but they were defined in experimental terms reminiscent of Paul Lazarsfeld's empirical work ${ }^{7}$ rather than as parts of robust theoretical frameworks. Influence, for example, if defined at all, was generally conceived as the capacity of an individual to change somebody else's 'opinion', as measured, for example, through voting in political elections. Even Kurt

7 Gitlin's (1978) critique of the 'dominant paradigm' in communications research can therefore be productively applied to sociometric concepts as well. 
Lewin explicitly acknowledged that the goal of mathematization overruled other considerations:

We have tried to avoid developing elaborate 'models'; instead we have tried to represent the dynamic relations between the psychological facts by mathematical constructs at a sufficient level of generality. (Lewin, 1964, p. 21)

And Kurt Lewin was certainly the sociometric scholar with the most substantial theoretical ambitions. Mullins (1973) squarely attributes the demise of small-group theory to the intellectual and theoretical vacuum left by Lewin's death in 1947. Granovetter's famous 'The Strength of Weak Ties' likewise ponders how '[s]ociometry, the precursor of network analysis, has always been curiously peripheral - invisible, really - in sociological theory' (Granovetter, 1973, p. 1360) and blames this not only on the difficulty to adapt methods to larger group sizes, but also on a lack of theoretical detail combined with 'a level of technical complexity appropriate to such forbidding sources as the Bulletin of Mathematical Biophysics' (Granovetter, 1973, p. 1361).

However, a glance at Granovetter's own work indicates where the field turned to find a new and more concise conceptual horizon: to social exchange theory, an approach that 'might be described, for simplicity, as the economic analysis of non economic social situations' (Emerson, 1976, p. 336). Here, power is no longer defined in vague psychological terms but in a serious, 'hard-knuckled' way as access to resources for economic advancement, to information on jobs, prices, opportunities, and so on. Since social exchange theory still relies on an atomistic and dyadic conception of social relations, it can accommodate graph theoretic models just as easily as attraction and repulsion did before. Having been turned into immutable mobiles by Harary and others, the mathematical concepts, methods, and metrics traveled effortlessly into the new conceptual space. ${ }^{8}$ What in Moreno's view had to be produced through 'spontaneity training', namely the free association of individuals, now conveniently becomes a basic property of individuals conceived as free agents engaged in the rational choice of utility maximization. ${ }^{9}$ While Mayer (2009) rightfully points to

8 As Emerson argues, social exchange theory is not a so much a theory, but 'a frame of reference within which many theories - some micro and some more macro - can speak to one another, whether in argument or in mutual support' (Emerson, 1976, p. 336).

9 'One answer, which we call "social atomism," suggests that social order is brought about through the free negotiations of autonomous individuals seeking to advance private interests. This view of social order is expressed in the 'social contract' theory associated with Hobbes, 
the sociometric heritage of contemporary search engine metrics and its closeness with cybernetics and operations research, I would argue that this economic trajectory constitutes an equally important lineage. It supplied a rich new space for conceptual inspiration, similar to the already mentioned input-output model, and it provided a new home for the mathematical metrics and methods pioneered in sociometry.

It is a core paradox of mathematical network analysis that it affords powerful tools for examining concrete power relations empirically, which holds considerable critical potential, while also allowing to apply the measured hierarchies as operative mechanisms to allocate resources such as visibility or research funding. What the different strands I have discussed here furnish - and this is fundamental for any form of normative and operational application of evaluative metrics, whether in citation ranking or in web search - is a narrative that sustains what could be called 'the innocence of the link': whether it is spontaneous attraction, rational choice, or simply an inspirational account of scientific citation as solely based on intellectual merit, the application of the metrics to actual ranking, with concrete and tangible consequences, can only be justified if the link is kept reasonably 'pure'. Like the theory of 'revealed preference' in the last chapter, these narratives seek to 'protect' the integrity of behavioral data as indicators for contested concepts such as relevance, authority, or impact. In this context, the main enemies are therefore the 'deceitful linkers', whether they come as scientific citation cartels or their contemporary cousins, link farms. It is not surprising that a central argument against citation analysis as a means for research evaluation builds on a critique of the purity of actual citation practices.

Despite these resemblances, we must recognize that there is not a singular blueprint underpinning formalization. Theoretical and methodological frameworks, grammatization through technical forms, or simply exploration and experimentation can inform the identification and demarcation of entities and relationships that become materials for algorithmic treatment. Ideas from various fields, from mathematics and engineering to the social sciences, economics, and business management, can inspire the operational makeup of these algorithmic procedures. And there is little reason to believe that commonsense reasoning, tacit knowledge, and learning from practice and experience cannot play a

Locke, Rousseau, Spencer, and others. Contemporary variations of social atomism include types of social psychological theory, especially economic-type exchange theory' (Lewis and Weigert, 1985, p. 455). 
central role in stirring the conceptual and technical imaginaries that inform technical creation.

So far, I have tried to sketch the historical background for certain core ideas present in PageRank in order to provide the material or 're-sources' (Chun, 2011, p. 20) for contextualizing this evaluative metric conceptually, in the sense that a critical reading of formulas or source code not only requires a capacity to understand technical languages but also interpretive ammunition to 'refill' computational artifacts that have most often been cleansed from easily accessible markers of context and origin. Historical investigation is indeed one way to reduce the opacity of software. If epistemological and methodological concepts such as the network model suggest a 'reading of reality' (Berry, 2008, p. 367), a historical approach can provide the material for an analysis of technicity that connects operation to a wider space of technical and nontechnical ideas. Such an analysis could seek to describe more general characteristics of the network approach, for example, the tendency to highlight 'the synchronic dispersal over the diachronic unfolding' (Berry, 2008, p. 367); it could situate it in relation to certain theories of the social and to specific mathematical methods, as I have tried to do in this section; but it could also attempt to home in further on technicity, which is the objective of the next section.

\section{Two Moments of Commitment}

To focus on the technical dimension of PageRank, I will develop two axes of mechanological interpretation, a comparative approach and a 'close reading' of a particular parameter, both organized around the question of how an understanding of metrics as 'descriptions' can help us apprehend how they become operative 'prescriptions' as engines of order. While citation metrics like the impact factor have quickly crossed the threshold between 'is' and 'ought', their use in 'science policy and research evaluation' (Garfield, 1972, p. 478 ) is hardly automated. Much like other statistical indicators before them, they have come to play an essential role in bureaucracies where ideals of so-called evidence-based governance privilege mathematical means of decision-making 'so constraining that the desires and biases of individuals are screened out', seeking to guarantee 'the rule of law, not of men' (Porter, 1995, p. 74). But even as integral parts of bureaucratic processes, these metrics retain both a certain visibility, which makes them amendable to critique, and margins of discretion in terms of how they are used. The rendering of graph theoretical methods in software is certainly significant 
on the descriptive side of things - just consider how SPSS ${ }^{10}$ plays as role in orienting research practices in the social sciences (Uprichard, Burrows, and Byrne, 2008) - but prescriptive power is particularly enhanced when metrics are sunk into the system and become both invisible and inevitable, in the sense that margins of discretion are defined by the system itself. This is how algorithmic techniques inform engines of order in a very direct sense, 'conducting conduct' (Foucault, 2008, p. 186) by means of modulating what can be seen and what can be done in an online environment. When using Google Search, neither the ranking principles, nor their parameters are amendable to user intervention. We may change the query and apply certain filters, but the way the result list is put into sequence remains out of our hand.

This begs the question of how the concepts and metrics outlined above found their way into computing and became part of how the web is ordered. In 1992, Botafogo, Rivlin, and Shneiderman first explored the transposition - or 'freezing' - of sociometric methods into a navigational software environment as a means to solve the 'lost in hyperspace' problem, that is, the disorientation users often experience when navigating in a hypertext. Building on a paper by Harary (1959), the authors formalized hypertexts as directed graphs and began calculating metrics for the purpose of 'recovering lost hierarchies and finding new ones' (Botafogo, Rivlin, and Shneiderman, 1992, p. 143). The goal was not to identify the most important nodes for document retrieval but rather to assist hypertext authors in designing the structure of their text networks more explicitly, based on the idea 'that structure does reflect semantic information' (p. 148). Hierarchies were seen as helpful navigational devices and status metrics would help building them into the hypertext's topology. Interestingly, Botafogo, Rivlin, and Shneiderman explicitly argued that software would make it easy to implement different network metrics and thereby provide more than one view on the hypertext, granting 'the ability to view knowledge from different perspectives' (p. 148). Once again, we encounter the idea that algorithmic information ordering can provide the means for 'fini-unlimited' permutation, where the latent structures present in the network are modulated and arranged at will.

Taking the work in sociometry, citation analysis, and hypertext navigation together, one could argue that all the 'ingredients' for PageRank were available from the middle of the 1990 and all one had to do was to combine them.

10 SPSS (formerly Statistical Package for the Social Sciences) is one of the most commonly used tools for statistical analysis in the social sciences. 
It is not the purpose of this chapter to judge the originality of Page and Brin's work but to show how network metrics were developed in different contexts and with different purposes in mind, and that they were indeed made operational in different ways and with different forms of performativity. This is where it is useful to connect back to Foucault's notion of the archive, understood not as a coherent 'paradigm' in Kuhn's (1962) sense, but as a wider 'conceptual field' (Foucault, 2002, p. 142) that allows for significant diversity and variation as well as regularity and 'transversal standardization' in the sense that similar metrics are applied in different domains.

The various metrics discussed over these last pages indeed define more general techniques for differentiation and distinction, but concrete calculative procedures and orchestrations can lead to very specific outputs. This means that an overarching critique of the reductionist and atomistic perspective espoused by the network model runs the risk of losing sight of the considerable margins for choice and expression available inside the conceptual space examined here. We therefore need to be attentive not only to moments of totalization, homogenization, and imposition but also to vectors of cumulative construction, heterogeneity, and fermentation. If algorithmic measurement increasingly means that 'authority no longer rests in the [social] relationships and instead migrates toward the measuring instrument' (Mayer, 2009, p. 68), the question of how we can better understand the way authority is concretely configured once it has migrated becomes virulent. Engaging PageRank as a model as well as an algorithmic technique can bring us closer to an answer. But this requires that we do more than capture its spirit; we have to examine its technical schema.

\section{Flatlands and Two Ways to Make Hills}

It is rather remarkable that, despite being initially conceived as a technique for finding scientific literature, citation analysis was not combined more thoroughly with existing techniques from information retrieval before the 1990s. The SCI's proprietary nature may have been a factor here, but it is safe to argue that the web, this gigantic and unruly mass of hyperlinked documents, played a significant role as catalyst in bringing together fields and methods that were largely separate until that point. Much like computerization and its different dimensions - including data availability, computing capability, and commercial opportunity - have informed the resurgence of information retrieval and its intermingling with artificial intelligence, the sheer size and complexity of the fledgling online spheres suggested that only algorithmic techniques would be capable of 'bringing order to the web' (Page et al., 1999). 
The first fifteen years of web history essentially play through the earlier 'information wars' in fast forward: manually assembled catalogs like the commercial Yahoo! Directory (closed in 2014) or the volunteer project DMOZ (closed in 2017) used elaborate hierarchical classification systems - or 'hierarchical ontologies' in contemporary vocabulary - to provide searchers with well-ordered maps covering the masses of documents. The criticism leveled against these efforts is virtually the same as almost 50 years earlier:

Human maintained lists cover popular topics effectively but are subjective, expensive to build and maintain, slow to improve, and cannot cover all esoteric topics. (Brin and Page, 1998, p. 117)

Automated search engines are presented as the answer, even if they have to deal with (partially) new problems, such as spamming and other forms of manipulation. Google Search has become almost synonymous with this automated approach. At first glance, it can be seen as a canonical information retrieval system that takes a query as input, searches through a (very large) document collection, and spits out a list of results. One could even make a legitimate comparison with coordinate indexing and argue that adding words to a query performs a Boolean AND and excluding words with the '-' operator a Boolean NOT. There is little reason to believe that Google's retrieval engine was initially all that different from the systems that Luhn and others had already experimented with in the late 1950s: word frequency counts, processing of document structure to identify 'privileged' text in titles or subheadings, statistical term-weighting schemes, and other techniques were well-known strategies for probabilistic, contentbased indexing and already commonly used in web search. Put simply, the shift in perspective associated with Google Search did not concern retrieval techniques as such but rather the way the collection itself was conceptualized.

Indeed, PageRank is sometimes presented as an example for the field of unsupervised machine learning (Hastie, Tibshirani, and Friedman, 2009, p. 576), a set of techniques briefly discussed in Chapter 5 that are often used to 'map out' a dataset, either through forms of classification or through attribution of some numerical value to individual items. In this case, PageRank is the mechanism by which the web is no longer treated exclusively as a document repository, but additionally as a social system that is riddled with the very 'socionomic hierarchies' Moreno described. By applying a recursive status index inspired by sociometry and citation analysis to the index of web pages, every document in the corpus can be 
located in the stratified network/society and ranked according to its 'status' before searching even begins. Attributing an 'importance score' (Page et al., 1999, p. 3) to every document 'independent of the page content' (Bianchini, Gori, and Scarselli, 2005, p. 94) fundamentally means giving up the idea of a 'flat corpus' (Chakrabarti, 2003) of documents where relevance is only determined in relation to a query. While information retrieval generally conceives relevance with regard to a specific 'informational need', the socio- and scientometric attributions of status, influence, or impact are much closer to forms of aggregate description like mapping or clustering. As an unsupervised machine learning technique that tries to find an optimal description for specific aspects of a dataset, PageRank establishes a global 'pecking order' for the web. More precisely, because linking is framed as a form of citation and thus as rational attribution of importance by document authors, the hyperlink network can become a singular, universal network of 'authority' that the search system can combine with traditional ranking mechanisms to calculate a final score during the search process. This quote from the initial research paper on PageRank highlights this two-prone approach:

The importance of a Web page is an inherently subjective matter, which depends on the readers interests, knowledge and attitudes. But there is still much that can be said objectively about the relative importance of Web pages. (Page et al., 1999, p. 1)

But how does this universalist component resonate with the larger argument for variation and perspectivism I have constructed over the last chapters? Comparing Google's algorithm to a close sibling points to moments of differentiation.

Just like PageRank, Jon Kleinberg's HITS (Hyperlink-Induced Topic Search) is an eigenvector-based evaluative metric that was introduced at nearly the same time as its more famous cousin (Kleinberg, 1999). Its virtually identical goal was to formulate 'a notion of authority' by processing the 'considerable amount of latent human judgment' encoded in hyperlinks, in order to solve the 'abundance problem' (p. 6o6) faced by web users. But there are two major differences.

First, HITS actually provides two eigenvector metrics, one for 'hubness' and another for 'authority'. Building on the idea 'that good hubs point to good authorities and good authorities are pointed to by good hubs' (Langville and Meyer, 2005, p. 136), Kleinberg makes a conceptual differentiation, entirely based on structural properties of the graph, resulting in a typology 
of nodes that, if implemented through an interface, could allow users to specify the type they are looking for. While metrics can indeed totalize and commensurate (Espeland and Stevens, 1998), there are many technical means to introduce differentiation and variation. In the domain of the fini-unlimited, one can not only calculate singular scores in countess ways but there is also no necessity to limit calculation to a singular score in the first place.

Second, HITS inverts the temporal sequence of authority ranking and document retrieval. Rather than calculate a universal or a priori landscape of authority like PageRank, documents matching a query are retrieved first and authority is calculated only second, taking only the link structure between found documents into account and not the full corpus. This means that in the HITS perspective, authority is dependent on a subject domain delineated through a query and a page that receives a high authority score for one search term may well get a much lower score for another. While this obviously does not abolish the notion of authority by any means, the concept is reconfigured, contextualized, and contained in the sense that HITS is less subject to what Barry and Browne (2006) euphemistically call 'topic drift' (p. 84), that is, the dominance of high-authority sites over a wide range of topics.

Algorithmic techniques do not exist in a disembodied state but become part of running systems where operations form (potentially recursive) sequences; simply rearranging these sequences may yield significant differences in terms of output. But being part of a running system also means that ideas compete on more than the merit of their results. Even if we may want to argue that the HITS perspective holds significant advantages over PageRank, the argument clashes with the fact that the computational requirements for HITS are much higher than for PageRank. While the latter can recalculate its universal landscape of authority at a set interval - before it fell silent on the topic, Google indicated a number of about once a month - the former would have to make an authority computation for every single search request. Certainly, the scores for the most popular queries could be calculated in advance, but a large disadvantage persists when it comes to both speed and cost. And the found documents for a given query may not even form a hyperlink network in the first place, reducing the system to traditional information retrieval metrics.

But even when looking inside the PageRank formula, we find space for variation and choice. In the next section, I will show how a single parameter encodes a significant theoretical and, consequently, operational commitment. 


\section{Objet petit $\alpha^{11}$}

Just like Katz's (1953) 'status index', PageRank not only 'recursively propagate[s] weights through the link structure of the Web' (Brin and Page, 1998, p. 108), but includes 'a damping factor that limits the extent to which a document's rank can be inherited by children documents' (Page, 2004, p. 7). The question is how far through the network authority should propagate. The rationale put forward by Page et al. (1999) to explain rank 'decay' (p. 4) in PageRank is twofold: first, the actual problem in the context of a web search engine is that without damping, a 'rank sink' in the form of a closed loop between two or more pages could excessively accumulate rank at each pass of the iterative method used to compute the metric, the usual approach to calculation in absence of an analytical solution. Damping eliminates those undesired artifacts. Second, an 'intuitive basis' (p. 5) in the form of the model of a random surfer is introduced, where the damping factor $\alpha$ 'models the fact that users typically jump to a different place in the web after following a few links' (Page, 2004, p. 7). While PageRank is presented as 'an attempt to see how good an approximation to "importance" can be obtained just from the link structure' (Page et al., 1999, p. 4), neither the 'rank sink' nor the 'random walk' justification really account for damping in respect to the question of importance. The first justification concerns a technical problem arising from the particular computational solution used and the second fails to elaborate how a model of random user behavior actually relates to the calculation of an importance score. While PageRank can indeed be read as a scoring of the 'reachability' of a node in a graph, in the sense that more central sites would be stumbled upon more frequently if somebody was randomly following links, the transfer from dampened reachability to importance remains unexplained. It is not surprising that Google nowadays prefers the term 'useful' to 'important'. ${ }^{12}$

Much like the admittedly difficult question of how language and meaning relate, which received surprisingly little attention in early information retrieval, the move from socio- and scientometrics to the web correlates with a dramatic loss of interest in considerations that could be described as 'domain theoretic'. These considerations, which inform and justify the

11 In Lacan's psychoanalysis, the 'objet petit a' stands for an object of desire that remains forever unattainable, elusive. Žižek (1992) argues that it 'is not a positive entity existing in space, it is ultimately nothing but a certain curvature of the space itself which causes us to make a bend precisely when we want to get directly at the object' (p. 48).

12 https://www.google.com/search/howsearchworks/algorithms/, accessed 22 February 2017. 
'appropriate coordination' between an empirical system and its formalization in the context of scientific description, are little more than inconveniences on the way toward computation. Terms like 'intuitive basis' signal the exchange of robust theoretical involvement for little more than commonsense reasoning, and this is really quite common in computing disciplines. We can, however, develop a line of mechanological interpretation that considers the explanations given by Katz and Bonacich for the inclusion of a damping factor into their own metrics.

Katz introduces the $\alpha$ factor as a means to adapt the calculation of his status index to 'both the group and the context' (Katz, 1953, p. 41) and provides an expressive example from the domain of information diffusion:

For example, the information that the new high-school principal is unmarried and handsome might occasion a violent reaction in a ladies' garden club and hardly a ripple of interest in a luncheon group of the local chamber of commerce. On the other hand, the luncheon group might be anything but apathetic in its response to information concerning a fractional change in credit buying restrictions announced by the federal government. (Katz, 1953, p. 41)

One way Katz (1953) interprets $\alpha$ is as an estimate of the 'probability of effectiveness of a single link' (p. 41) for relaying information and he argues that this is highly dependent on context and, in particular, on the fit between the social setting and the message in question. In this sense, $\alpha$ encodes the probability that a piece of information is passed on from one person to the next and a researcher should choose an appropriate level based on her appreciation of the situation. A high level of damping means that information will not spread very far, even if high status individuals are involved. The 'conductivity' is considered to be low and status does not reach very far into the network. In contrast, if one looks at a military command structure, for example, conductivity can be considered to be very high since orders are generally followed from top to bottom. In this case, status would be strongly cumulative since a powerful individual like a general has full control over what happens further down the network hierarchy. If $\alpha$ is small, control over others is considered low and status ranking actually approaches the 'balloting' Katz criticized as dissatisfying, because status no longer propagates and the simple number of votes a person receives becomes the determining factor. Because already small differences in the factor can lead to significant variation in outcomes, the commitment to a particular estimation of the conductivity of a network - we must not forget that $\alpha$ is 
a manually set constant - has substantial consequences on the calculated hierarchies. Indeed, Bianchini, Gori, and Scarselli discuss $\alpha$ in PageRank in similar terms as the factor deciding how far the structure of a network should influence the status of a node:

PageRank deeply depends on parameter $[\alpha]$. When $[\alpha]$ is small, only the nearest relatives give an actual contribution to [PageRank], whereas, as $[\alpha] \rightarrow 1$, far ancestors are also significant for the evaluation of [PageRank]. (Bianchini, Gori, and Scarselli, 2005, p. 125)

The conceptual entanglements this modulation implies clears up further when examining the arguments behind Bonacich's (1987) decision to add a damping factor to his own 'power centrality' metric. In order to account for the results of an empirical study by Cook et al. (1983), which had shown a disjunction between network centrality and power in a bargaining network, Bonacich proposes a parameter $\beta$, which regulates the depth of influence in a very similar fashion to $\alpha$ by defining 'a radius within which power or centrality is being assessed' (Bonacich, 1987, p. 1174). What makes Bonacich's piece so interesting is his reflection on how $\beta$ becomes a way to implement varying conceptions of what constitutes power in different social networks. The full quote is instructive:

To some, the measure may seem hopelessly ambiguous; [power centrality] can give radically different rankings on centrality, depending on the value of $\beta$. However, the measure accentuates an inherent ambiguity in the concept of centrality. There are different types of centrality, depending on the degrees to which local and global structures should be weighted in a particular study and whether that weight should be positive or negative. When communication is typically over long distances, position in the global structure should count more than when all communication is local. In an organized hierarchy in which power is transitive, the power of those one has power over should be weighted more highly in determining overall power than when all relations are dyadic. (Bonacich, 1987, p. 1181)

We notice again that a single parameter is seen as encoding a fundamental appreciation of how influence or power operate in a given situation. Bonacich's $\beta$ actually goes beyond $\alpha$, because it is designed to work with negative values as well, so that connections to high status nodes are actually detrimental to rank. This allows the model to account for bargaining networks, where exchange theory considers it beneficial to be connected 
to a large number of low status nodes that can be more easily exploited because they lack information, for example, about prices. In this situation, 'power comes from being connected to those who are powerless' (p. 1171). Because $\beta$ and $\alpha$ are set manually, they express a true commitment to a theory about the real-world properties of the network in question, even if that commitment is made with little consideration for its implications.

When applying this discussion to PageRank, two very different notions of status, authority, and importance emerge: with $\alpha \rightarrow 0$, the web is indeed conceived as a 'one person, one vote' type democracy ${ }^{13}$ and collecting incoming links, no matter from where, would be the principal means of gaining rank; in a $\alpha \rightarrow 1$ setting, the web becomes a very different place - opaque, complex, and potentially Machiavellian, where success comes from either patronage by the already powerful or the patient and costly construction of large and spread-out strategic networks. While we have to keep in mind that this is a scale and not a binary decision, the first vision puts an emphasis on the local properties of the graph and makes it therefore possible for local changes, if significant in number, to affect the overall ranking of the system; the second builds on the global structure of the graph and local changes therefore have negligible effects on overall rankings. If we keep with the image of a social system, the respective models of transformation are 'populist/revolutionary' and 'conservative/reformatory'. While purely descriptive metrics make an epistemological commitment to a theory of power to represent social status, the prescriptive mode of PageRank as an engine of order in a technical system makes an operational commitment to reproduce and reinforce, in very practical terms, the hierarchy of social status it detects. The value Google usually communicated for $\alpha$ was 0.85 (Brin and Page, 1998, p. 110) and at this level, the often-heard interpretation of PageRank as essentially an expression of popularity (Diaz, 2008; Rieder, 2009) misses the target. While sheer numbers certainly do play a role, the idea that PageRank interprets the web as 'a gigantic popularity contest' (Roush, 2004, n.p.) does not capture how it 'brings order to the web'. At a level of 0.85 , power is highly cumulative and an inlink from a high-status node will be much more valuable than from a site with lower status.

If we consider Google Search as a central site of power negotiation and arbitrage for the web, an analysis focusing on PageRank, which in practice

13 Whether voting is necessarily a core principle of democratic government is debatable, however. As Bernard Manin (1997) has shown, up to the French revolution, elections were generally associated with aristocracy and selection by lottery was seen as the truly democratic mode of assigning office. 


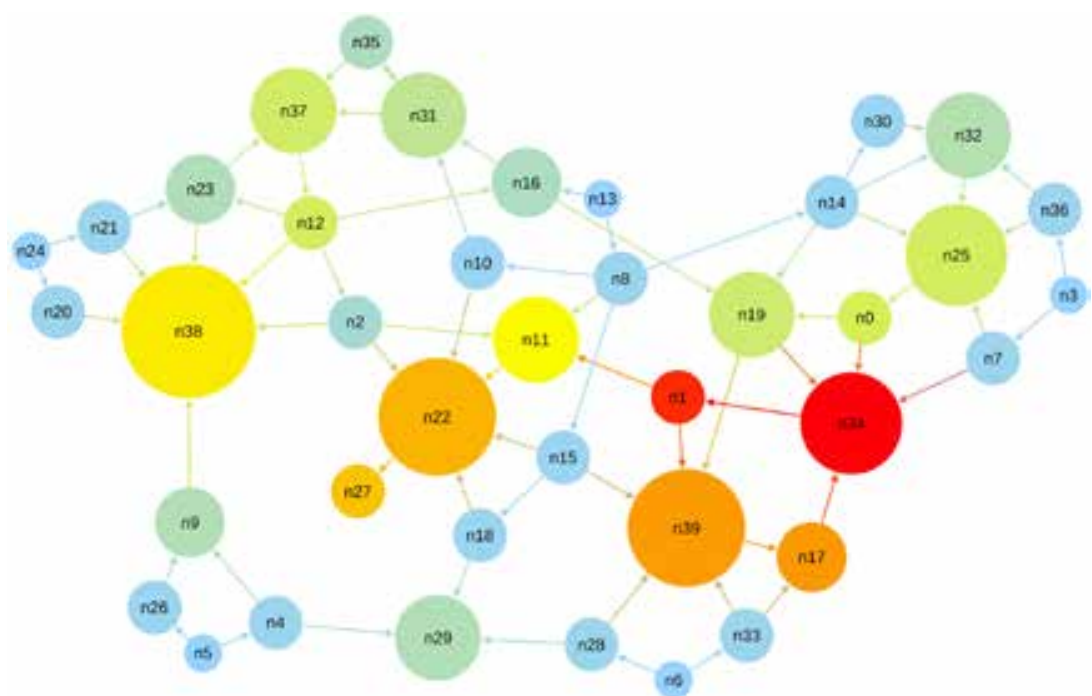

A network generated randomly with Gephi. Node sizes represent the number of inlinks and color represents PageRank $(a=0.85$ ), going from light to dark grey. We instantly notice that $\mathrm{n} 1$ has a high PR although it only receives a single inlink. This link comes from the highest status node (n34), however, and since that node links nowhere else, $\mathrm{n} 1$ receives a lot (85 percent) of its status.

\begin{tabular}{llllllll}
\hline Label & PR $(a=0.85)$ & $P R(a=0.7)$ & $P R(a=0.55)$ & $P R(a=0.4)$ & In-Degree & Out-Degree & Degree \\
\hline n34 & 0.0944 & 0.0743 & 0.0584 & 0.0460 & 4 & 1 & 5 \\
n1 & 0.0867 & 0.0617 & 0.0450 & 0.0345 & 1 & 2 & 3 \\
n17 & 0.0668 & 0.0521 & 0.0423 & 0.0355 & 2 & 1 & 3 \\
n39 & 0.0663 & 0.0541 & 0.0453 & 0.0388 & 5 & 1 & 6 \\
n22 & 0.0619 & 0.0506 & 0.0441 & 0.0393 & 5 & 1 & 6 \\
n27 & 0.0591 & 0.0451 & 0.0371 & 0.0318 & 1 & 0 & 1 \\
n38 & 0.0522 & 0.0561 & 0.0542 & 0.0486 & 6 & 0 & 6 \\
n11 & 0.0492 & 0.0372 & 0.0306 & 0.0274 & 3 & 1 & 4 \\
\hline
\end{tabular}

A table of eight nodes from the same network as above, selected and ordered based on the highest values for PageRank $(a=0.85)$. Four PageRank rankings were calculated. Note how $n 1$ with just a single inlinks slips with lower values for $a$ and $n 38$ rises to the top of the ranking.

is certainly insufficient, would have to conclude that its authority-ranking mechanism applies, in a universal fashion, a largely conservative ${ }^{14}$ vision of society to the document graph, one that picks out 'the real leaders' (Katz,

14 The term is used here in a rather literal sense as favoring the maintenance of the status quo. The difficulty to apply traditional concepts from political theory becomes evident when we take into account that the 'revolutionary masses' PageRank is set to defend against are mostly spammers and search engine optimizers. 
1953, p. 39) and distributes visibility to them. Rather than showing us the popular it shows us the authoritative, or, to connect back to citation analysis, the canonical (Introna, 2007, p. 18). If we consider the link indeed as 'innocent', as a valid indicator of disinterested human judgment, PageRank shows us a meritocracy; if we take the link to be fully caught up in economic forces, however, we receive the map of a plutocracy. In either case, the search engine as an engine of order will accentuate the difference between high and low rank over time since pages with higher status will be more visible and thus have a greater chance of receiving further links.

Similar to the Bayesian classifier, computer scientists have studied the effects of $\alpha$ in great detail (Langville and Meyer, 2004, 2005; Bianchini, Gori, and Scarselli, 2005). But the above figure and table show the effects of changes in the value for $\alpha$ on a simple and actually rather 'egalitarian' network in a hopefully more accessible form than the papers written by specialists. While we cannot make any far-reaching generalizations based on a limited experiment, it is in line with the general finding that small variations in $\alpha$ can lead to significant changes in ranking. It also confirms that at high values for $\alpha$ the quality of inlinks is much more relevant than their number; a single link from a page with a high rank can be extremely valuable, especially if that page does not link anywhere else. When lowering $\alpha$ and thus adding more damping, the hierarchy shifts in favor of nodes with many inlinks. Although this is only a sketch, approaches involving tinkering or 'screwing around' (Ramsay, 2010) with algorithms should be considered as ways of studying them as 'beings that function' (Simondon, 2017, p. 151) in their natural habitat: eating numbers and spewing out different numbers at the rear end.

But again, computational requirements add an interesting caveat. While the level set for $\alpha$ has important conceptual and potentially political consequences, it also implies a cost factor, since a higher value means that the calculation will require a larger number of iterations to converge. ${ }^{15}$ Langville and Meyer capture the dilemma:

Consequently, Google engineers are forced to perform a delicate balancing act. The smaller $\alpha$ is, the faster the convergence, but the smaller $\alpha$ is, the less the true hyperlink structure of the web is used to determine webpage importance. And slightly different values for $\alpha$ can produce very different PageRanks. (Langville and Meyer, 2004, p. 346)

15 In the iterative power method for calculating PageRank, only an approximation to the eigenvector solution is calculated. The loop stops when the changes from one iteration to the next fall below a set level. 
This means that a local theory of power is computationally and, because computation costs money, economically cheaper than a theory where status is based on global structural properties. In this case, the menace from spam apparently appeared as sufficiently substantial to convince Google that a larger radius of influence - and consequently a reduced capacity for local changes to affect global structure - was well worth the additional cost.

\section{Variation and Combination}

This chapter set out to examine what is 'in' the PageRank algorithm and it proceeded in two ways: It first situated this evaluative metric in a larger archive of statements, that is, of ideas, concepts, theories, methods, and techniques that developed in and around sociometry, citation analysis, social exchange theory, and hypertext navigation. This conceptual space constitutes, at least to a certain extent, the place of emergence from where PageRank became 'sayable' (cf. Foucault, 2002, p. 146). The fact that many of the papers discussed here are cited in the relevant patents adds concreteness and plausibility to this idea. The chapter deviated from the preceding ones by taking a contemporary technique as its narrative telos. Although an implicit focus on the present has guided my selections in earlier examples as well, there is a more specific reason for this choice: while information retrieval revolves around the conceptual and operational possibilities of computing machinery from the beginning and takes earlier work in information ordering mainly as negative inspiration, PageRank epitomizes a moment when a long and illustrious trajectory of calculative techniques finds a place and raison d'être in an information infrastructure supported by networked computing. Certainly, both sociometry and citation analysis were using computing machinery from the 1960 s on and the latter, in particular, has been operating as an engine of order entangled in research evaluation and resource allocation almost from the beginning. But the shift from these 'ancestral' domains into document retrieval and ranking is much more abrupt that the rather steady accumulation of techniques discussed in previous chapters. These differences between trajectories indicate that algorithmic techniques are characterized by variation not only in terms of technicity and conceptual content, but also in terms of invention and evolution. If ' $[\mathrm{w}]$ hat resides in the machines is human reality, human gesture fixed and crystallized in working structures' (Simondon, 2017, p. 18), there is no single way this 'fixing and crystallizing' happens, no canonical sequence of steps, and no singular discipline or materiality that holds everything 
together. PageRank illustrates how a heterogeneous archive of statements and practices finds expression in the particular form of an algorithmic technique, becoming ready-to-hand not only for software developers that want to use it in their own programs, but also for users engaged in the mapping practices mentioned at the beginning of this chapter: using a graph analysis toolkit like Gephi, PageRank becomes applicable to any network at the click of a button. Unsurprisingly, it asks its users to set a value for $\alpha$, somewhere between 0 and 1 .

Second, by comparing the algorithm to a close cousin and by examining a particular parameter in the model, the chapter tried to show that the concrete schema - the algorithmic technique, not the search engine - does not simply follow from the historical a priori. There are thousands of graph theoretical algorithms documented and hundreds of variants of the recursive status metrics I have focused on. Are they all based on a reductionist and atomistic vision of social relations? Quite possibly. Do they all encode the same logic, produce the same rankings, and commit to the same politics? Certainly not. There is variation and it is significant: even a single parameter can have extraordinary consequences for the actual ordering performed.

While my discussion of PageRank was more technical than earlier examples, the goal was to show how deeply entangled with social and cultural questions technicity can become, in particular when let loose into concrete application settings. Although Larry Page's proposition is basically just a way to transform one set of numbers into another set, what is actually expressed are not only mathematical functions but quite fundamental ideas about authority, importance, relevance, and so forth. Technicity, indeed, draws on different forms of imagination, even if the expression as a technical idea requires a capacity to function. The language used may be that of mathematics and code, but the expressed technical schema captures the outside world and, by becoming operational as part of a search engine, shows us that world in particular ways. Information retrieval's reluctance to formulate more elaborate theories of language, meaning, or knowledge does not prevent actual systems to enact operational interpretations of these things, simply by doing what they do. The orderings most of us are confronted with several times a day when querying Google Search are as much deliberate outcomes as the tree of knowledge Diderot and D'Alembert organized the Encyclopédie around. But the ways in which these outcomes come about could not be more different.

Like the techniques discussed in earlier chapters, network algorithms start from a common way of representing data as a graph, an intermediate form that implies a particular formalization of the empirical realities they 
target. While the feature vector 'sees' the world as sets of objects having certain properties, the graph frames objects in terms of their connections. We find a similar capacity for generalization and generativity: if a phenomenon can be fitted into an adjacency matrix, a large number of readily available techniques can be applied at will. While this chapter was mainly concerned with the various ways hierarchies of authority, influence, status, and so forth can be measured, this is only one of many things network algorithms can be used for. One could write a similar story for the detection of socalled 'cliques', for example, densely connected groups of friends in social networks, scientific disciplines or paradigms in citation networks, or sets of products that are bought together frequently. Or a story covering the various measures of 'betweenness' (Freeman, 1977) that seek to detect nodes that are 'bridging' ${ }^{\prime 16}$ subgroups and can therefore not only access a potentially wider set of resources but also control the information flow between different parts of the network. The excitement around a science of networks that attempts to consider common aspects of networks across domains is indeed nourished by the fact that so many different phenomena can be productively modeled as graphs. We thus find the same ordering techniques applied to phenomena ranging from molecular interactions to collaborations between movie actors. But in all of these cases, mathematical transformations are coordinated with domain-specific interpretations in some way and the same metric will not necessarily express the same idea when applied to two different types of network, opening a space for interpretation and critique.

It should come as no surprise that the graph and the feature vector are not competing or mutually exclusive paradigmatic forms, but 'merely' pivotal instances in malleable spaces of technical expression. Ultimately, there is parallelism and abundant potential for connection in the field of algorithmic techniques and moving from one form or technique to another often requires little more than a change in perspective. The hyperlink network does not have to be treated strictly as a graph. We could, for example, consider a web document as a feature vector comprised not of words, but of the entities it links to. Akin to Henry Small's co-link analysis, we could then use Salton's similarity calculations to find pages that are 'related' because they link to similar places but are not directly connected. But the statistical technique does not really tell us what 'related' would actually mean here. Would we

16 Putnam (2000) distinguishes between two types of social capital: bonding capital is high for individuals that are central in a tightly knit group; bridging capital is high for those that connect disparate groups or circles. Many different algorithms have tried to formalize and calculate these ideas. 
be able to find a theoretical framework to support and justify our idea or would we simply create and evaluate an experimental setup to demonstrate the capacity and utility of our system? Combining trajectories in the other direction, we could follow Luhn's idea and formalize co-occurrences of words in a text as a graph, submitting the resulting network to any number of centrality metrics, in the hope of finding the most 'important' words in the document. This technique, co-word analysis, is indeed quite common and we may rightfully ask what kind of 'capture' of language and meaning it implies. The functional forms of signification such operations may deploy in specific settings is not culturally neutral but also not easily aligned with cultural categories and dependent on the data under scrutiny.

While these are somewhat arbitrary examples, technical plasticity can be engaged more proactively and with a critical mindset. Connecting back to the discussion of software-making in Chapter 3 , we can recognize that the plasticity software affords is not distributed equally, turning it into a site of political struggle. An example from my own work can illustrate what I mean. Almost fifteen years ago, I called for developer access to Google's search index, based on the idea that the enormous trust users place in the company should give rise to a 'symmetry of confidence' (Rieder, 2005) in the form of increased openness to (programmed) interaction with its products and infrastructures. To give concrete meaning to this proposal, I built a small web mashup on the basis of Yahoo!'s now defunct BOSS (Build your own Search Service) Web API. TermCloud Search would get 500 search results, each pretagged with a set of keywords, and then generate a word cloud based on these keywords. The cloud was meant to break with the linear hierarchy of the result list, to map the result space by providing an overview, and to furnish a visual representation that could be used to navigate the results by clicking on words. It was a basically a coordinate indexing system sitting on top of ranked search results. Multiple terms could be selected to create an AND query and the tool used simple keyword co-occurrence to show which terms appeared most commonly together.

This rather basic example is meant to show how engaging technicity does not require a deep dive into mathematical principles but may well begin with attentiveness to broad techniques, end-user functionalities, and interface arrangements. TermCloud Search can be understood as an attempt at critical contribution, in the form of an object, to the debate around the specific rationales information retrieval systems should implement. While Google Search generally emphasizes the capacity to deliver the right 'answer' to a 'question' in the least amount of time, my mashup considered the query not so much as a question, but as a way to evoke a potentially 


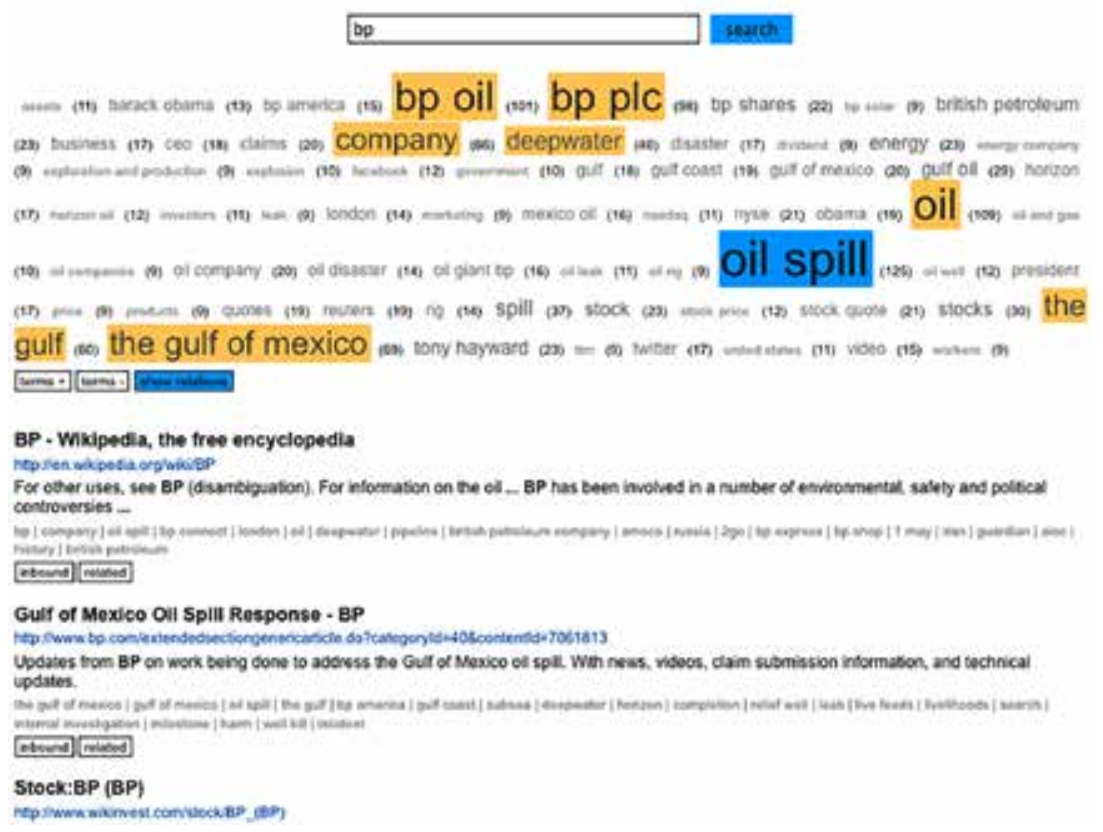

The interface of termCloud Search, showing results for the query [bp]. Clicking on the term 'oil spill' would show the results tagged with that term and highlight the terms that co-occurred most often with it.

multifaceted or contested topic, and the result not as an answer, but as a crude overview and navigational interface into the different dimensions that topic may have. While this certainly overstates the conceptual reach of such a small experiment, one could say that the design objective was to induce a sensitivity for the plurality and composite character of an issue as well as to practically facilitate its exploration.

While the playful combination of techniques becomes clearly visible in this example, (almost) all software is a hybrid when looking behind the interface, even if design goals can certainly be less benign. Could we imagine a machine learning setup that sets $\alpha$ in PageRank dynamically, based on feedback, in order to delegate the inconvenient moment of human judgment and theoretical commitment to an empirical machine? If our goal is to deliver the 'best' results, we could very well calculate PageRank at different levels of $\alpha$ and submit each level to a randomly selected subset of our user base. In line with the notion of revealed preference, we may take it as a positive signal when a user actually clicks on the first link. If users select lower ranked links, we take this as negative feedback. The level of $\alpha$ with 
the highest percentage of positive signals wins and the problem is solved. Imagine similar games not for a single instance of algorithmic ordering, but for many: some concerning the formalization of document content, some concerning link structure, some concerning implicit or explicit feedback; others slicing users by location, by device form factor, or by movement speed in physical space; yet others personalizing based on use history or similarity with other users. If we imagine this huge network of possible factors and techniques - and probably a more complex appreciation of what success means - and we end up with a more accurate, albeit still superficial, appreciation of how systems like Google Search actually work and evolve over time. Google almost certainly scores on the basis of some intricate and adaptive combination of relevance (information retrieval), authority (network analysis), and feedback (machine learning) techniques, combining the different technical trajectories discussed in this book into a complex technical ensemble. Because each of the technical elements in play is complex and their (possible) interactions even more so, thousands of people with PhDs work at Google, not merely a few. As Peter Norvig, now Director of Research at Google, has recently argued, 'the competitive advantage really comes from the hard work of what you do with the algorithm and all the processes around making a product, not from the core algorithm itself' (r/Science and AAAS-AMA, 2018, n.p.). Technology is created from technical elements, but their arrangement and connection with the world increasingly relies on empirical processes that tie design, use, and evaluation tightly together into an object that is never entirely finished and therefore never fully 'detaches' from its producer the way Simondon describes (Simondon, 2014, p. 27). The study of algorithmic techniques gives us a handle on the vocabulary of functional expression, but the way technology is actually 'spoken' is increasingly hard to decipher.

\section{Bibliography}

Barabási, A.-L. (2002). Linked: The New Science of Networks. Cambridge: Perseus Publishing.

Barnes, J. A., and Harary, F. (1983). Graph Theory in Network Analysis. Social Networks 5(2), 235-244.

Bastian, M., Heymann, S., Jacomy, M. (2009). Gephi: An Open Source Software for Exploring and Manipulating Networks. In International AAAI Conference on Weblogs and Social Media. Retrieved from https://www.aaai.org/ocs/index. php/ICWSM/og/paper/view/154. 
Bavelas, A. (1948). A Mathematical Model for Groups Structures. Human Organization $7(3), 16-30$.

Benkler, Y, (2006). The Wealth of Networks: How Social Production Transforms Markets and Freedom, New Haven: Yale University Press.

Berry, D. M. (2008). The Poverty of Networks. Theory, Culture and Society 25(7-8), 364-372.

Berry, M. W., and Browne, M. (2006). Understanding Search Engines: Mathematical Modeling and Text Retrieval ( $2^{\text {nd }}$ ed.). Philadelphia: Society for Industrial and Applied Mathematics.

Bianchini, M., Gori, M., and Scarselli, F. (2005). Inside PageRank. ACM Transactions on Internet Technology 5(1), 92-128.

Bonacich, P. (1972). Factoring and Weighting Approaches to Status Scores and Clique Identification. Journal of Mathematical Sociology 2(1), 113-120.

Bonacich, P. (1987). Power and Centrality: A Family of Measures. American Journal of Sociology 92(5), 1170-1182.

Botafogo, R. A., Rivlin, E., and Shneiderman, B. (1992). Structural Analysis of Hypertexts: Identifying Hierarchies and Useful Metrics. ACM Transactions on Information Systems 1o(2), 142-180.

Brin, S., and Page, L. (1998). The Anatomy of a Large-Scale Hypertextual Web Search Engine. Computer Networks and ISDN Systems 30, 107-117.

Burrows, R. (2012). Living with the H-Index? Metric Assemblages in the Contemporary Academy. Sociological Review 6o(2), 355-372.

Castells, M. (1996). The Information Age: Economy, Society and Culture, Volume 1: The Rise of the Network Society. Cambridge, Oxford: Blackwell.

Chakrabarti, S. (2003). Mining the Web: Discovering Knowledge from Hypertext Data. San Francisco: Morgan-Kauffman.

Chun, W. H. K. (2011). Programmed Visions. Cambridge, MA: MIT Press.

Cook, K. S., Emerson, R. M., Gilmore, M. R., and Yamagishi, T. (1983). The Distribution of Power in Exchange Networks: Theory and Experimental Results. American Journal of Sociology 89(2), 275-305.

Diaz, A. (2008). Through the Google Goggles: Sociopolitical Bias in Search Engine Design. In A. Spink and M. Zimmer (eds.), Web Search:Multidisciplinary Perspectives (pp. 11-34). Berlin: Springer.

Emerson, R. M. (1976). Social Exchange Theory. Annual Review of Sociology 2, 335-362.

Erdős, P. (1977). A Note of Welcome. Journal of Graph Theory 1(3), 3.

Espeland, W. N., and Stevens, M. L. (1998). Commensuration as a Social Process. Annual Review of Sociology 24, 313-343.

Festinger, L. (1949). The Analysis of Sociograms using Matrix Algebra. Human Relations 2(2), 153-158. 
Forsyth, E., and Katz, L. (1946). A Matrix Approach to the Analysis of Sociometric Data: Preliminary Report. Sociometry 9(4), 340-347.

Foucault, M. (2002). Archaeology of Knowledge (A. M. Sheridan Smith, trans.). London: Routledge.

Freeman, L. C. (1977). A Set of Measures of Centrality Based on Betweenness. Sociometry $40(1), 35-41$.

Garfield, E. (1955). Citation Indexes for Science: A New Dimension in Documentation through Association of Ideas. Science 122(3159), 108-111.

Garfield, E. (1963). Science Citation Index. In E. Garfield (ed.), Science Citation Index 1961 (pp. v-xvi). Philadelphia: Institute for Scientific Information.

Garfield, E. (1972). Citation Analysis as a Tool in Journal Evaluation. Science $178(4060), 471-479$.

Gießmann, S. (2009). Ganz klein, ganz groß. Jacob Levy Moreno und die Geschicke des Netzwerkdiagramms. In I. Köster and K. Schuster (eds.), Medien in Zeit und Raum. Maßverhältnisse des Medialen (pp. 267-292). Bielefeld: transcript Verlag.

Gitlin, T. (1978). Media Sociology: The Dominant Paradigm. Theory and Society $6(2), 205-253$.

Granovetter, M. S. (1973). The Strength of Weak Ties. American Journal of Sociology 78(6), 1360-1380.

Gross, P. L. K., and Gross, E. M. (1927). College Libraries and Chemical Education. Science 66(1713), 385-389.

Hacking, I. (1990). The Taming of Chance. Cambridge: Cambridge University Press. Hanneman, R. A., and Riddle, M. (2005). Introduction to Social Network Methods. Riverside: University of California, Riverside. Retrieved from http://faculty.ucr. edu/ hanneman/.

Harary, F. (1959). Status and Contrastatus. Sociometry 22(1), 23-43.

Harary, F. (1969). Graph Theory. Reading: Addison-Wesley.

Harary, F. (1983). Conditional Connectivity. Networks 13(3), 347-357.

Harary, F., and Norman, R. Z. (1953). Graph Theory as a Mathematical Model in Social Science. Ann Arbor: Institute for Social Research, University of Michigan.

Harary, F., Norman, R. Z., and Cartwright, D. (1965). Structural Models: An Introduction to the Theory of Directed Graphs. New York: John Wiley \& Sons.

Hastie, T., Tibshirani, R., and Friedman, J. H. (2009). The Elements of Statistical Learning: Data Mining, Inference, and Prediction ( $2^{\text {nd }}$ ed.). New York: Springer.

Hubbell, C. H. (1965). An Input-Output Approach to Clique Identification. Sociometry 28(4), 377-399.

Introna, L. D. (2007). Maintaining the Reversibility of Foldings: Making the Ethics (Politics) of Information Technology Visible. Ethics and Information Technology $9(1), 11-25$. 
Katz, L. (1953). A New Status Index Derived from Sociometric Analysis. Psychometrika $18(1)$, 39-43.

Kleinberg, J. M. (1999). Authoritative Sources in a Hyperlinked Environment. Journal of the ACM 46(5), 604-632.

Kőnig, D. (1936). Theorie der endlichen und unendlichen Graphen. New York: Chelsea.

Kuhn, T. (1962). The Structure of Scientific Revolutions. Chicago: University of Chicago Press.

Langville, A. N., and Meyer, C. D. (2004). Deeper Inside PageRank. Internet Mathematics 1(3), 335-380.

Langville, A. N., and Meyer, C. D. (2005). A Survey of Eigenvector Methods for Web Information Retrieval. SIAM Review 47(1), 135-161.

Latour, B. (1986). Visualization and Cognition: Thinking with Eyes and Hands. Knowledge and Society: Studies in the Sociology of Culture Past and Present 6, 1-40.

Lewin, K. (1936). Principles of Topological Psychology. New York: McGraw-Hill.

Lewin, K. (1964). Field Theory in Social Science: Selected Theoretical Papers. New York: Harper \& Row.

Lewis, D., and Weigert, A. J. (1985). Social Atomism, Holism, and Trust. Sociological Quarterly 26(4), 455-471.

Manin, B. (1997). The Principles of Representative Government. Cambridge: Cambridge University Press.

Mayer, K. (2009). On the Sociometry of Search Engines: A Historical Review of Methods. In K. Becker and F. Stalder (eds.), Deep Search: The Politics of Search beyond Google (pp. 54-72). Piscataway: Transaction Publishers.

Mirowski, P., and Nik-Khah, E. (2017). The Knowledge We Have Lost in Information. Oxford: Oxford University Press.

Moreno, J. L. (1934). Who Shall Survive? A New Approach to the Problem of Human Interrelation. Washington, DC: Nervous and Mental Disease Publishing.

Mullins, N. C. (1973). Theories and Theory Groups in Contemporary American Sociology. New York: Harper \& Row.

Page, L. (2001). Method for Node Ranking in a Hyperlinked Database. US Patent 6,285,999, filed 9 January 1998 and issued 4 September 2001.

Page, L. (2004). Method for Scoring Documents in a Linked Database. US Patent 6,799,176, filed 6 July 2001 and issued 28 September 2004.

Page, L., Brin, S., Motwani, R., and Winograd, T. (1999). The PageRank Citation Ranking: Bringing Order to the Web [technical report]. Stanford InfoLab. Retrieved from http://ilpubs.stanford.edu:809o/422/.

Pinski, G., and Narin, F. (1976). Citation Influence for Journal Aggregates of Scientific Publications. Information Processing and Management 12(5), 297-312. 
Porter, T. (1995). Trust in Numbers: The Pursuit of Objectivity in Science and Public Life. Princeton: Princeton University Press.

Putnam, R. D. (200o). Bowling Alone. New York: Simon and Schuster.

r/Science, and AAAS-AMA. (2018). AAAS AMA: Hi, we're researchers from Google, Microsoft, and Facebook who study Artificial Intelligence. Ask us anything! The Winnower. https://doi.org/10.15200/winn.151896.65484.

Ramsay, S. (2010). The Hermeneutics of Screwing Around; or What You Do with a Million Books. In K. Kee (ed.), Pastplay: Teaching and Learning History with Technology (pp. 111-120). Ann Arbor: University of Michigan Press.

Rieder, B. (2005). Networked Control: Search Engines and the Symmetry of Confidence. International Review of Information Ethics 3, 26-32.

Rieder, B. (2009). Democratizing Search? From Critique to Society-Oriented Design. In K. Becker and F. Stalder (eds.), Deep Search: The Politics of Search beyond Google (pp. 133-151). Piscataway: Transaction Publishers.

Rieder, B. (2013) Studying Facebook via Data Extraction: The Netvizz Application. In H. Davis, H. Halpin, and A. Pentland (eds.), WebSci '13 Proceedings of the $5^{\text {th }}$ Annual ACM Web Science Conference (pp. 346-355). New York: ACM.

Rogers, R. (2013). Digital Methods. Cambridge, MA: MIT Press.

Roush, W. (2004). Search beyond Google. MIT Technology Review, 1 March. Retrieved from https://www.technologyreview.com.

Salton, G. (1963). Associative Document Retrieval Techniques Using Bibliographic Information. Journal of the ACM 10 (4), 440-457.

Shore, C., and Wright, S. (2000). Coercive Accountability: The Rise of Audit Culture in Higher Education. In M. Strathern (ed.), Audit Cultures: Anthropological Studies in Accountability, Ethics and the Academy (pp. 57-89). London: Routledge. Simondon, G. (2014). Sur la technique (1953-1983). Paris: Presses Universitaires de France.

Simondon, G. (2017). On the Mode of Existence of Technical Objects (C. Malaspina and J. Rogove, trans.). Minneapolis: Univocal Publishing.

Small, H. (1973). Co-citation in the Scientific Literature: A New Measure of the Relationship between Two Documents. Journal of the American Society for Information Science 24(4), 265-269.

Stigler, S. M. (1999). Statistics on the Table: The History of Statistical Concepts and Methods. Cambridge, MA: Harvard University Press.

Topîrceanu, A. (2017). Breaking Up Friendships in Exams: A Case Study for Minimizing Student Cheating in Higher Education Using Social Network Analysis. Computers \& Education 115,171-187.

Uprichard, E., Burrows, R., and Byrne, D. (2008). SPSS as an 'Inscription Device': From Causality to Description? Sociological Review 56(4), 6o6-622. 
Watts, D. J. (2004). The 'New' Science of Networks. Annual Review of Sociology zo, 243-270.

Zimmer, M. (2010). Web Search Studies: Multidisciplinary Perspectives on Web Search Engines. In J. Hunsinger, L. Klastrup, and M. Allen (eds.), International Handbook of Internet Research (pp. 507-521). Dordrecht: Springer.

Žižek, S. (1992). Enjoy Your Symptom! Jacques Lacan in Hollywood and Out. New York: Routledge. 